\section{Effect of OCT volume scan density on thickness measurements in diabetic macular edema}

MG Nittala, R Konduru, H Ruiz-Garcia

and SR Sadda
DME. Manual correction of segmentation errors appeared to have a clinically meaningful effect in a small minority of cases. These results may have implications for the design of SD-OCT imaging and grading protocols in clinical trials of DME, particularly when using multiple SD-OCT instruments that acquire varying numbers of B-scans. Eye (2011) 25, 1347-1355; doi:10.1038/eye.2011.173; published online 15 July 2011

Keywords: optical coherence tomography; retinal thickness; diabetic macular edema; scanning density

\section{Introduction}

With its capacity to provide detailed visualization of retinal morphology, optical coherence tomography (OCT) ${ }^{1}$ has rapidly become an integral part of retinal clinical practice. ${ }^{2,3}$ Major applications of OCT in ophthalmology include identifying vitreomacular interface abnormalities, 3,4 monitoring features of exudation in the setting of choroidal neovascularization, and assessing the morphology and quantifying the extent of macular edema. ${ }^{5}$ This ability to provide quantitative information, in particular measures of macular thickness, is perhaps one of OCT's most important applications. ${ }^{3}$

Measurement of retinal thickness by the OCT instrument software depends upon accurate identification of the internal limiting membrane and the hyper-reflective band that is believed to represent the outer border of the retina. ${ }^{3}$ With Stratus OCT (a time domain OCT instrument, Carl Zeiss Meditec, Dublin, CA, USA) this outer retinal border was mistakenly placed at the band that is now believed to correspond to the
The Department of Ophthalmology, Doheny Eye Institute and Keck School of Medicine of the University of Southern California, Los Angeles, CA, USA

Correspondence: SR Sadda, The Department of Ophthalmology, Doheny Eye Institute and Keck School of Medicine of the University of Southern California, Los Angeles, CA 90033, USA Tel: + 1323442 6503; Fax: + 13234426460 . E-mail: ssadda@ doheny.org

Received: 8 April 2011 Accepted in revised form: 30 May 2011 Published online: 15 July 2011 
photoreceptor inner segment-outer segment junction. With the advent of spectral domain OCT technologies, this outer border has been moved posteriorly to a more anatomically correct position, although the exact position varies between spectral domain OCT (SD-OCT) instruments (Carl Zeiss Meditec). Regardless of the precise position of the outer retinal boundary, in all instruments, the OCT software calculates the distance between inner and outer retinal boundary of the retina to estimate the retinal thickness. But questions have been raised about the accuracy and reliability of OCT retinal thickness measurements. ${ }^{3,4,6}$ These measurements can be affected by artifacts, ${ }^{6,7}$ poor signal, ${ }^{5}$ operator errors, decentration errors due to poor fixation and failure of the OCT software to accurately segment the retinal boundaries. ${ }^{3}$ Segmentation errors are particularly frequent in pathological conditions, where disruption of the normal retinal bands can present challenges to automated alogrithms. ${ }^{3,8,9}$ Sadda $e t \mathrm{al}^{3}$ found that retinal thickness measurement errors may be observed in $92 \%$ of eyes imaged with Stratus OCT. Glassman et $a l^{10}$ reported, however, that manual correction of errors in Stratus OCT-automated analysis appeared to have no appreciable effect on the interpretation of anatomic outcomes for diabetic macular edema (DME).

In contrast, SD-OCT instruments offer dramatically higher image acquisition speeds, allowing denser, more complete coverage of the macula with raster scans. ${ }^{11,12}$ Despite the improved acquisition speeds and advances in image processing offered by SD-OCT, segmentation errors still frequently occur. Keane et $a l^{13}$ noted retinal thickness measurement errors in $57.5 \%$ of eyes imaged with the Cirrus high-definition OCT (HD-OCT, Carl Zeiss Meditec), with severe errors occurring in $9.6 \%$ of these eyes. The impact of manual correction of automated segmentation errors on dense SD-OCT datasets has not been carefully studied, in part because correction of errors in large numbers of B-scans may be exhaustive and impractical for routine use. In addition, the effects of segmentation errors on global parameters such as total macular volume (TMV) have not been well studied. We have demonstrated that only a small subset of B-scans in a volume scan may be necessary to generate an accurate retinal thickness and volume map. ${ }^{14}$ However, this previous study included various retinal pathologies and was not powered to determine the minimum required scanning density for specific diseases such as diabetic macular edema. In addition, the previous study included only cases without segmentation errors and thus was not designed to evaluate the impact of correcting these segmentation errors.

In this report, we evaluate the impact of reducing the density of B-scans and manual correction of segmentation errors on SD-OCT retinal thickness maps in patients with DME.

\section{Materials and methods}

In all, 64 eyes of 43 consecutive patients with macular edema secondary to diabetic retinopathy were enrolled in this retrospective study. All 64 eyes had undergone Cirrus HD-OCT (Carl Zeiss Meditec) imaging. The study was approved by the institutional review board of the University of Southern California, and the research adhered to the tenets set forth in the Declaration of Helsinki. Other patient variables, including gender, age, best-corrected visual acuity, systemic and concurrent ocular disease, and ophthalmoscopic and biomicroscopic findings, were also culled from the patient records.

All patients referred to the Doheny Ophthalmic Imaging Unit for Cirrus OCT imaging undergo a standardized macular cube protocol consisting of 128 equally spaced, horizontally oriented, $6 \mathrm{~mm}$ raster B-scans, each composed of 512 A-scans, with scanning performed within a $6 \mathrm{~mm}$ square centered on the fovea. This protocol is used rather than the $200 \times 200$ cube protocol, because the $512 \times 128$ protocol is the preferred and accepted protocol at image-reading centers for clinical trials and because the ophthalmologists at our institution have found the $6 \mathrm{~mm}$ B-scans composed of only 200 A-scans to be of insufficient quality for detailed morphological assessment. For this study, raw OCT scan data were exported from the Cirrus OCT and then imported into previously described and validated SD-OCT-reading center grading software known as 3D-OCTOR (created by Doheny Image Reading Center software engineers, Los Angeles, CA, USA). ${ }^{14,15}$

\section{Generating retinal thickness maps}

Retinal thickness maps were regenerated using 3D-OCTOR to evaluate the relationship between retinal thickness measurements and B-scan-sampling density. A total of six different, evenly spaced B-scan densities were evaluated: 1) all 128 B-scans (spaced $47 \mu \mathrm{m}$ apart); 2) 64 B-scans, (every other scan, $94 \mu \mathrm{m}$ apart); 3) 32 B-scans (every fourth scan, $188 \mu \mathrm{m}$ apart); 4) 16 B-scans (every eighth scan, $376 \mu \mathrm{m}$ apart); 5) 8 B-scans (every sixteenth scan, $752 \mu \mathrm{m}$ apart); 6) 4 B-scans (every thirty-second scan, $1504 \mu \mathrm{m}$ apart). The first scan at the top of the cube was the starting scan for 128 B-scan cube, the second scan from the top was the starting scan for the 64 (every other) B-scan cube, the fourth scan from the top was the starting scan for the 32 (every fourth) B-scan cube, and so forth. Measurements for each of the ETDRS subfields and the TMV (volume of all nine subfields combined) were automatically computed for each sampling density using a simple bilinear interpolation as previously described. ${ }^{14}$ In addition, a trained, certified reading center grader (MGN) performed manual correction of all B-scans in all 

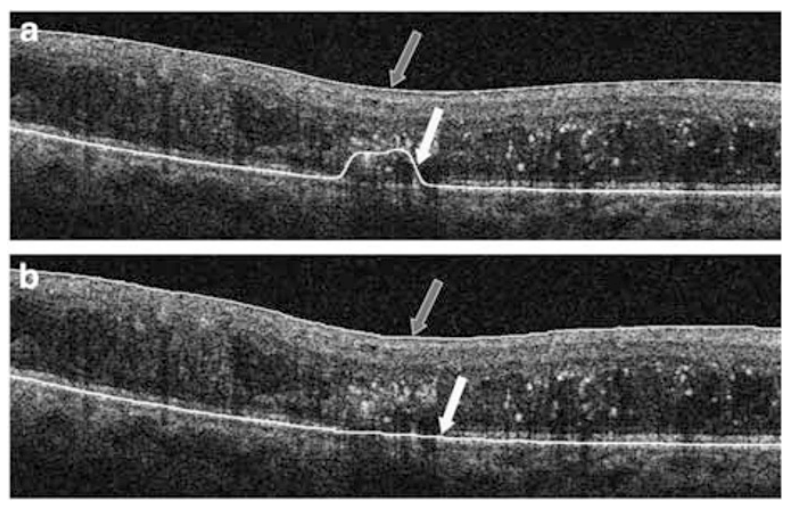

Figure 1 (a) Uncorrected optical coherence tomography (OCT) B-scan with retinal segmentation boundaries generated by the Cirrus OCT instrument software. (b) Corrected OCT B-scan with manual placement of retinal boundaries; blue arrow indicates the inner limiting membrane boundary and the white arrow indicates the retinal pigment epithelial boundary. The color reproduction of this figure is available at the Eye Journal online.

cases that demonstrated any error in segmentation of the inner or outer retinal boundaries (Figure 1). The reproducibility of reading center correction of retinal boundaries has been demonstrated in previous reports. ${ }^{16}$ Retinal thickness maps were regenerated for each sampling density after manual correction (using bilinear interpolation).

To determine whether regional variations in the extent of edema influenced the optimal OCT scanning density, retinal thickness values (generated using the corrected 128 B-scans) for each subfield were then compared with normative thickness values provided from previous publications of Cirrus OCT imaging in normal, healthy individuals. ${ }^{17}$ In accordance with previous DRCR.net publications, subfields with mean thickness values $>2$ standard deviations above the mean normal value for that subfield were deemed to be thickened. ${ }^{17}$ The number of thickened subfields (maximum of nine) was determined for each case and was used as a measure to determine if the edema was focal (fewer thickened subfield) or diffuse (more thickened subfields).

\section{Statistical methods}

For statistical analysis and comparison, retinal thickness and volume measurements derived from using all 128 B-scans were considered the corrected or uncorrected reference standard, depending on whether manual correction of segmentation errors had been performed. The corrected reference standard was considered to be the ground truth. The difference (or error) between the uncorrected reference standard value for the foveal central subfield (FCS) and the TMV was calculated for each scanning density for each case. This analysis of varying B-scan densities was also repeated after all segmentation errors were corrected, and corrected measurements were also compared with uncorrected values. Best-corrected visual acuity was converted to log MAR notation for statistical analysis. Absolute difference and percentage errors were calculated. Bland-Altman plots were generated to facilitate the comparison between values for each B-scan density and the reference standard measurements. Intra-class correlation analysis was performed to study the relationship between reference standard measurements and thickness measurements at various frame-sampling densities. Linear regression analysis was used to evaluate the relationship between number of thickened subfields and absolute difference in thickness measurements at varying frame-sampling densities. All data were analyzed using statistical software SPSS 15.0 (SPSS, Chicago, IL, USA) and MedCalc (MedCalc Software, ver. 11.3.8, Mariakerke, Belgium). A $P$-value of $\geq 0.05$ was considered statistically significant. One-way ANOVA and Bonferroni correction were used to determine significant differences between and within B-scan densities.

\section{Results}

A total of 64 eyes with DME from 43 patients were included in the study. The mean age of included patients was $62.1 \pm 8.9$ (SD) years, with a median age of 62.5 years. The mean duration of diabetes was $20.1 \pm 9.2$ years, and the mean best-corrected visual acuity was 0.44 (Snellen $\approx 20 / 50) \pm 0.55$. Increasing FCS retinal thickness, as determined using all 128 B-scans, was associated with reduced visual acuity $(r=0.299, P=0.016)$.

\section{B-scan frame-sampling density and retinal thickness}

Table 1 shows the absolute difference and percentage error of retinal thickness in both corrected and uncorrected sampling groups. Using scans without manual correction of segmentation errors, the mean absolute error (relative to 128 uncorrected B-scans) at the FCS was $0.11 \pm 0.08 \mu \mathrm{m}$ with 64 B-scans ( 1 of 2 ), increasing to $7.43 \pm 6.25 \mu \mathrm{m}$ with $8 \mathrm{~B}$-scans. The percentage error was $0.04 \pm 0.03 \%$ with 64 B-scans (1 of 2), increasing to $2.39 \pm 2.16 \%$ with 8 B-scans. There was no statistically significant difference between the absolute differences with sampling densities of 128, 64, 32 , or 16 B-scans. However, a statistically significant difference $(P=0.001)$ in FCS thickness relative to that obtained with 128 B-scans was observed at sampling densities of 8 B-scans or lower.

Following recalculation of all retinal thickness maps after correction of retinal boundary errors, the mean 
Table 1 Absolute difference and percentage error of retinal thickness in both corrected and uncorrected sampling groups

\begin{tabular}{|c|c|c|c|c|c|c|c|}
\hline \multicolumn{4}{|c|}{ Corrected scan sampling } & \multicolumn{4}{|c|}{ Uncorrected scan sampling } \\
\hline Scan density & Mean & $S D$ & Range & Scan density & Mean & $S D$ & Range \\
\hline \multicolumn{4}{|c|}{ Absolute difference $(\mu \mathrm{m})$} & \multicolumn{4}{|c|}{ Absolute difference $(\mu \mathrm{m})$} \\
\hline 64 & 0.29 & 0.47 & $0-2.4$ & 64 & 0.11 & 0.08 & $0-0.3$ \\
\hline 32 & 1.11 & 1.53 & $0-7.6$ & 32 & 0.57 & 0.47 & $0-1.9$ \\
\hline 16 & 2.43 & 1.88 & $0-10.1$ & 16 & 2.21 & 1.65 & $0-8.7$ \\
\hline 8 & 7.71 & 6.30 & $0.3-28.7$ & 8 & 7.43 & 6.25 & $0.3-29.9$ \\
\hline 4 & 15.41 & 28.24 & $0-220$ & 4 & 11.91 & 11.20 & $0-48.3$ \\
\hline \multicolumn{4}{|c|}{ Percentage error (\%) } & \multicolumn{4}{|c|}{ Percentage error (\%) } \\
\hline 64 & 0.09 & 0.14 & $0-0.75$ & 64 & 0.04 & 0.03 & $0-0.12$ \\
\hline 32 & 0.34 & 0.44 & $0-2.38$ & 32 & 0.19 & 0.19 & $0-1.01$ \\
\hline 16 & 0.79 & 0.70 & $0-4.09$ & 16 & 0.70 & 0.55 & $0-2.17$ \\
\hline 8 & 2.31 & 1.78 & $0.11-8.39$ & 8 & 2.39 & 2.16 & $0.09-11.16$ \\
\hline 4 & 4.75 & 8.73 & $0-67.92$ & 4 & 3.87 & 4.10 & $0-16.66$ \\
\hline
\end{tabular}

Scan density $=$ number of B scans.

absolute error (relative to the corrected ground truth 128 B-scans) at the FCS was $0.29 \pm 0.49 \mu \mathrm{m}$ with $64 \mathrm{~B}$-scans and $7.71 \pm 6.3 \mu \mathrm{m}$ with $8 \mathrm{~B}$-scans. The percentage error was $0.09 \pm 0.14 \%$ with $64 \mathrm{~B}$-scans, increasing to $2.31 \pm 1.78 \%$ with 8 B-scans. Figure 2 shows the comparative graph of absolute error and percentage error of FCS retinal thickness for the various sampling densities. Bland-Altman plots of the mean difference in FCS thickness between the ground truth and each reduced sampling density are shown in Figure 3. The mean neurosensory FCS retinal thicknesses at the varying densities were $345.17 \pm 107.97 \mu \mathrm{m}$ (with 128 B-scans), $345.22 \pm 107.77 \mu \mathrm{m}$ (64 B-scans), $345.02 \pm 107.14 \mu \mathrm{m}$ (32 B-scans), $345.97 \pm 106.19 \mu \mathrm{m}$ (16 B-scans), $345.26 \pm 100.89 \mu \mathrm{m}$ (8 B-scans), and $336.63 \pm 102.94 \mu \mathrm{m}$ (4 B-scans). Increasing ground truth (128 corrected B-scans) FCS thickness showed a statistically significant negative correlation (ie smaller error) with percentage error at sampling densities of 64 B-scans $(r=-0.249, P=0.04), 32 \mathrm{~B}$-scans $(r=-0.333$, $P=0.007), 16$ B-scans $(r=-0.563, P=0.001)$, and 8 B-scans $(r=-0.247, P=0.05)$, but was not statistically significant for 4 B-scans $(r=-0.145, P=0.252)$. The ICC coefficient was 1.00 (95\% CI: 1.00-1.00) for a framesampling density of 64 B-scans and 0.978 (0.930-0.973) for a frame-sampling density of 4 B-scans.

\section{B-scan-sampling density and TMV}

Table 2 shows the absolute difference and percentage error of TMV in both corrected and uncorrected sampling groups. For scans without manual correction of segmentation errors, the mean absolute difference in TMV was $0.001 \pm 0.0032 \mathrm{~mm} 3$ for 64 B-scans ( 1 of 2),
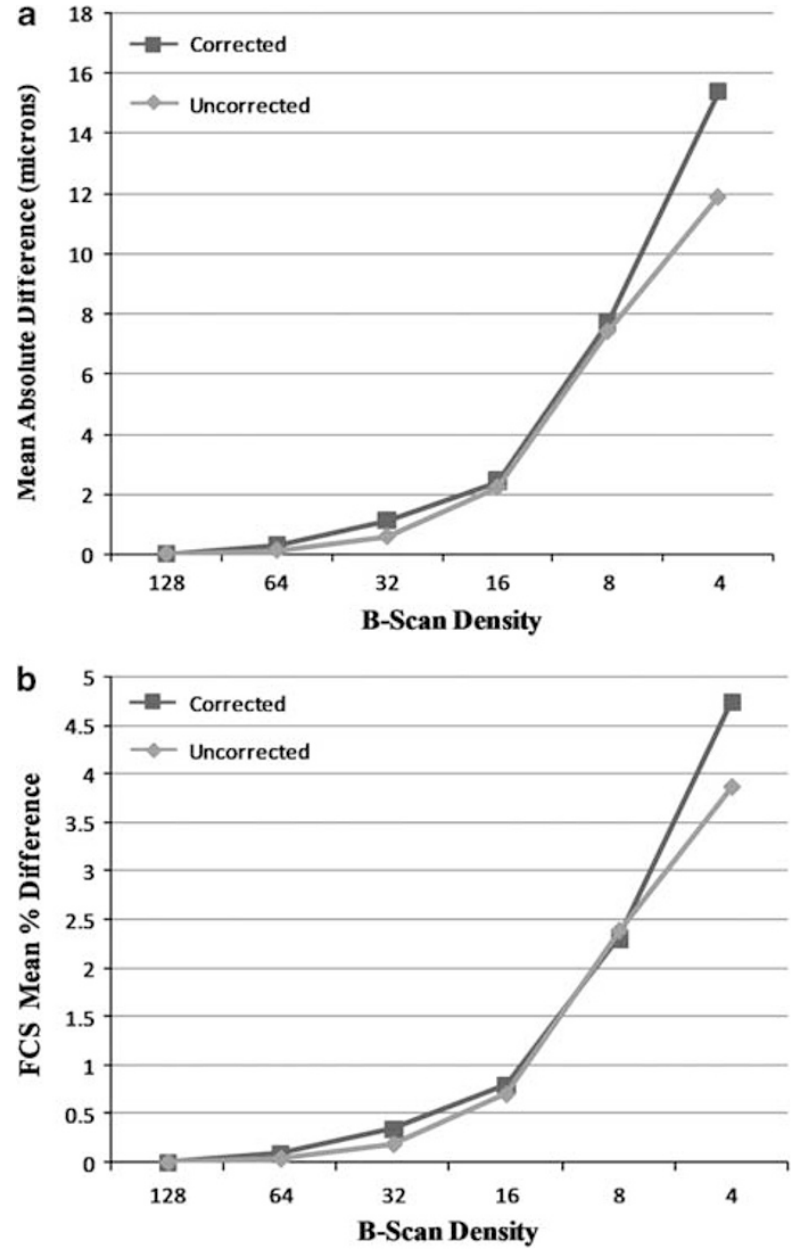

Figure 2 Effect of reduced B-scan density on measurements of foveal central subfield (FCS) thickness of corrected versus uncorrected retinal segmentation boundaries: (a) mean absolute difference $(\mu \mathrm{m}),(b)$ mean percentage error. 

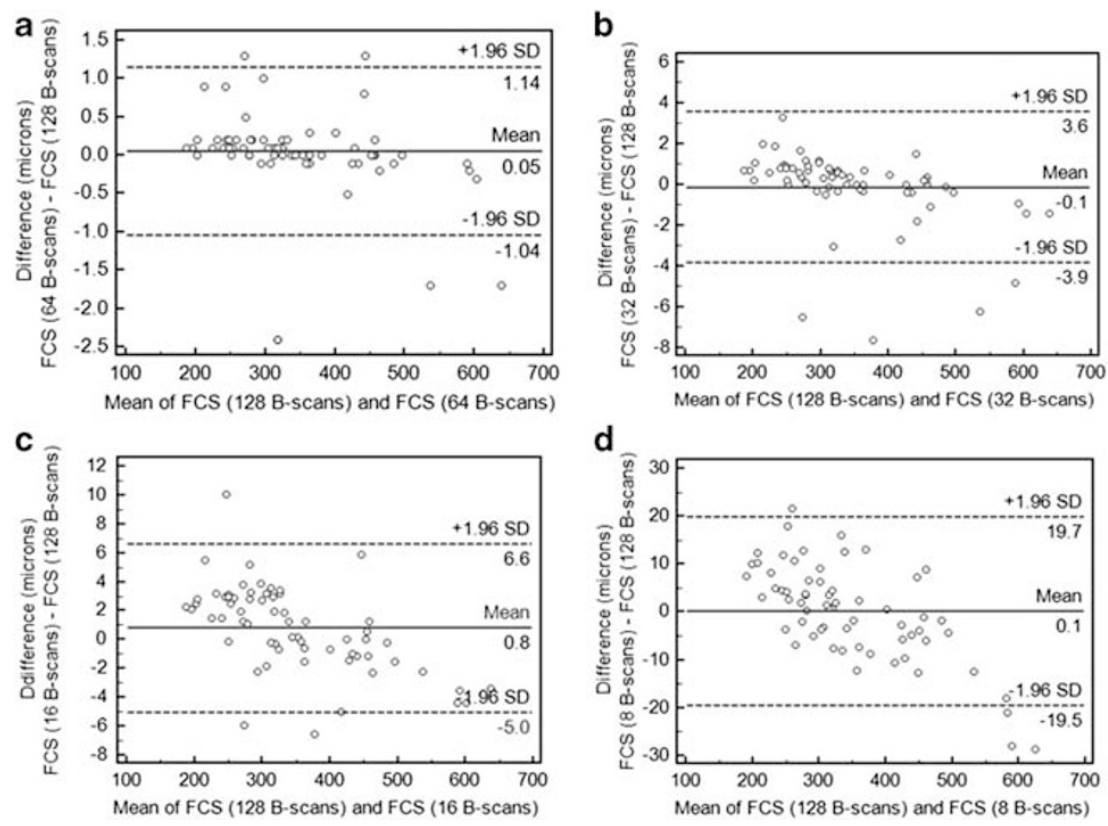

Figure 3 Bland-Altman plots demonstrating the level of agreement between the ground truth and each reduced sampling density for the foveal central subfield retinal thickness. Central solid line indicates the mean absolute difference and dotted lines indicate the $95 \%$ confidence interval limits (upper and lower). (a) At a B-scan-sampling density that includes every other B-scan (64 B-scans with an equal spacing of $94 \mu \mathrm{m}$ ). (b) At a B-scan-sampling density that includes every fourth B-scan (32 B-scans with an equal spacing of $188 \mu \mathrm{m}$ ). (c) At a B-scan-sampling density that includes every eighth B-scan (16 B-scans with an equal spacing of $376 \mu \mathrm{m}$ ). (d) At a B-scan-sampling density that includes every sixteenth B-scan (eight B-scans with an equal spacing of $752 \mu \mathrm{m}$ ).

Table 2 Absolute difference and percentage error of total macular volume in both corrected and uncorrected sampling groups

\begin{tabular}{|c|c|c|c|c|c|c|c|}
\hline \multicolumn{4}{|c|}{ Corrected scan sampling } & \multicolumn{4}{|c|}{ Uncorrected scan sampling } \\
\hline Scan density & Mean & $S D$ & Range & Scan density & Mean & $S D$ & Range \\
\hline \multicolumn{4}{|c|}{ Absolute difference $\left(\mathrm{mm}^{3}\right)$} & \multicolumn{4}{|c|}{ Absolute difference $\left(\mathrm{mm}^{3}\right)$} \\
\hline 64 & 0.003 & 0.005 & $0-0.02$ & 64 & 0.001 & 0.003 & $0-0.01$ \\
\hline 32 & 0.007 & 0.009 & $0-0.04$ & 32 & 0.003 & 0.005 & $0-0.01$ \\
\hline 16 & 0.012 & 0.010 & $0-0.04$ & 16 & 0.010 & 0.009 & $0-0.04$ \\
\hline 8 & 0.028 & 0.020 & $0-0.09$ & 8 & 0.023 & 0.018 & $0-0.07$ \\
\hline 4 & 0.117 & 0.219 & $0-1.72$ & 4 & 0.089 & 0.072 & $0-0.36$ \\
\hline \multicolumn{4}{|c|}{ Percentage error (\%) } & \multicolumn{4}{|c|}{ Percentage error (\%) } \\
\hline 64 & 0.03 & 0.05 & $0-0.19$ & 64 & 0.01 & 0.04 & $0-0.14$ \\
\hline 32 & 0.07 & 0.10 & $0-0.47$ & 32 & 0.04 & 0.05 & $0-0.14$ \\
\hline 16 & 0.13 & 0.10 & $0-0.47$ & 16 & 0.11 & 0.11 & $0-0.55$ \\
\hline 8 & 0.31 & 0.22 & $0-0.95$ & 8 & 0.25 & 0.20 & $0-0.75$ \\
\hline 4 & 1.33 & 2.77 & $0-22.14$ & 4 & 0.98 & 0.79 & $0-3.9$ \\
\hline
\end{tabular}

Scan density $=$ number of $B$ scans.

rising to $0.0023 \pm 0.02 \mathrm{~mm}^{3}$ with 8 B-scans. The percentage error was $0.013 \pm 0.04 \%$ with 64 B-scans, increasing to $0.25 \pm 0.2 \%$ with 8 B-scans. There was no statistically significant difference between the absolute differences for all frame-sampling densities (128 B-scans-4 B-scans).

Following recalculation of all retinal thickness maps after correction of retinal boundary errors, the mean absolute difference in TMV was $0.003 \pm 0.005 \mathrm{~mm}^{3}$ with $64 \mathrm{~B}$-scans, rising to $0.028 \pm 0.02 \mathrm{~mm}^{3}$ with $8 \mathrm{~B}$-scans. The percentage error was $0.03 \pm 0.05 \%$ with $64 \mathrm{~B}$-scans, increasing to $0.31 \pm 0.22 \%$ with $8 \mathrm{~B}$-scans. There was no statistically significant difference between the absolute differences for all frame-sampling densities when compared with the ground truth value. Figure 4 shows the comparative graph of absolute error and percentage 

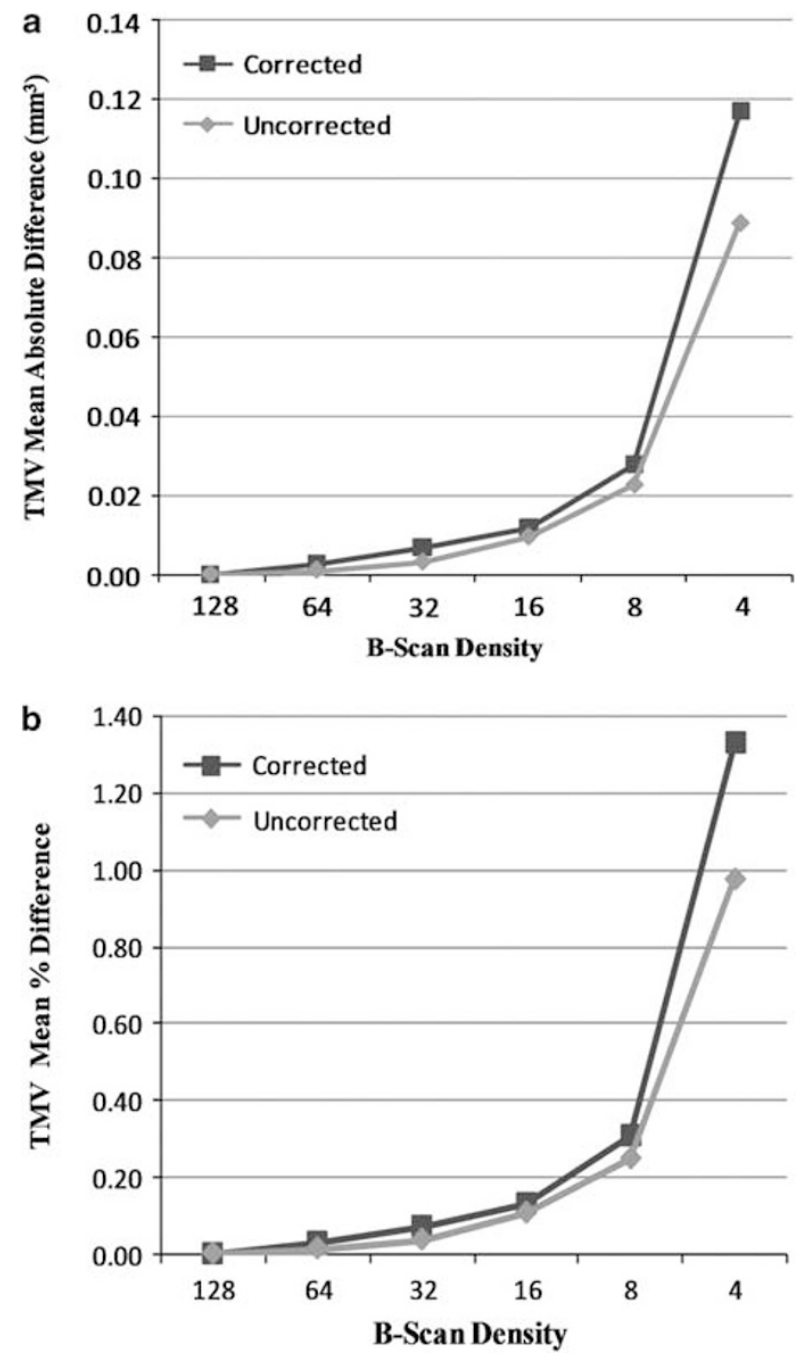

Figure 4 Effect of reduced B-scan-sampling density on measurements of total macular volume of corrected versus uncorrected retinal segmentation boundaries: (a) mean absolute difference $\left(\mathrm{mm}^{3}\right),(b)$ mean percentage error.

error of TMV for all sampling densities. For TMV, Bland-Altman plots of the mean difference between the ground truth and each sampling density are shown in Figure 5. The ICC coefficient was 1.00 (95\% CI: 1.00-1.00) for a frame-sampling density of 64 B-scans and 0.998 $(0.995-0.996)$ at the lowest frame-sampling density of 4 B-scans.

\section{Corrected versus uncorrected thickness and volume}

Table 3 compares retinal thickness and volume of the corrected and uncorrected sampling groups. Manual correction of retinal boundaries in at least one B-scan was necessary in 35 of the 64 eyes $(54.7 \%)$, but the differences were not statistically significant for the overall group.
However, $12.5 \%$ of eyes did demonstrate a difference of more than $10 \mu \mathrm{m}$ between corrected and uncorrected scans, with a maximum difference of $115.7 \mu \mathrm{m}$ in one case. The frequency distribution of thickness measurement differences between corrected and uncorrected scans is shown in Figure 6.

The TMV observations were similar to results of FCS retinal thickness measurements, with no statistically significant difference between corrected and uncorrected groups at all scanning densities (128 B-scans-4 B-scans). We also found no significant correlation between the number of thickened subfields (a measure of the extent of the macular edema) and the absolute difference in thickness measurements relative to the ground truth for any sampling density $(r=0.047 ; P=0.715$ at framesampling density of $16 \mathrm{~B}$-scans).

\section{Discussion}

In this study, we aimed to evaluate the effect of reducing B-scan density and correcting segmentation errors on retinal thickness measurements in eyes with diabetic macular edema. Our results suggest that retinal thickness and volume maps similar to those obtained using the dense 128 B-scan macular cube can be generated with a subset of as few as every eighth scan (16 B-scans with equal spacing of $376 \mu \mathrm{m}$ ), both for FCS thickness and for TMV. At a scanning density of every eighth scan (16 B-scans) the maximum error in FCS retinal thickness for any eye was $4 \%$.

Thus, it appears that for patients with DME, the minimum number of scans required is fewer than was concluded to be necessary in prior studies that included patients with a variety of different diseases. ${ }^{14}$ A potential explanation for this observation is that eyes with diabetic macular edema may have more gradual changes in topology than the eyes of patients with diseases such as neovascular AMD. Thus, a smaller sampling of the macula may adequately represent the extent and severity of the disease. Interestingly, we observed no relationship between the number of subfields thickened by edema (a measure of diffuseness), and the error in thickness measurement.

Regardless, these findings may have implications for the design of OCT acquisition and grading protocols for clinical trials relating to diabetic macular edema. With respect to acquisition protocols, some SD-OCT

instruments that employ tracking and extensive B-scan averaging may not be able to acquire very dense volume cubes in a clinically practical time frame because of poor fixation or other patient factors. If multiple SD-OCT instruments are to be used within a particular trial, it is reassuring to observe that varying scan densities can still yield fairly comparable results for DME. With respect to 

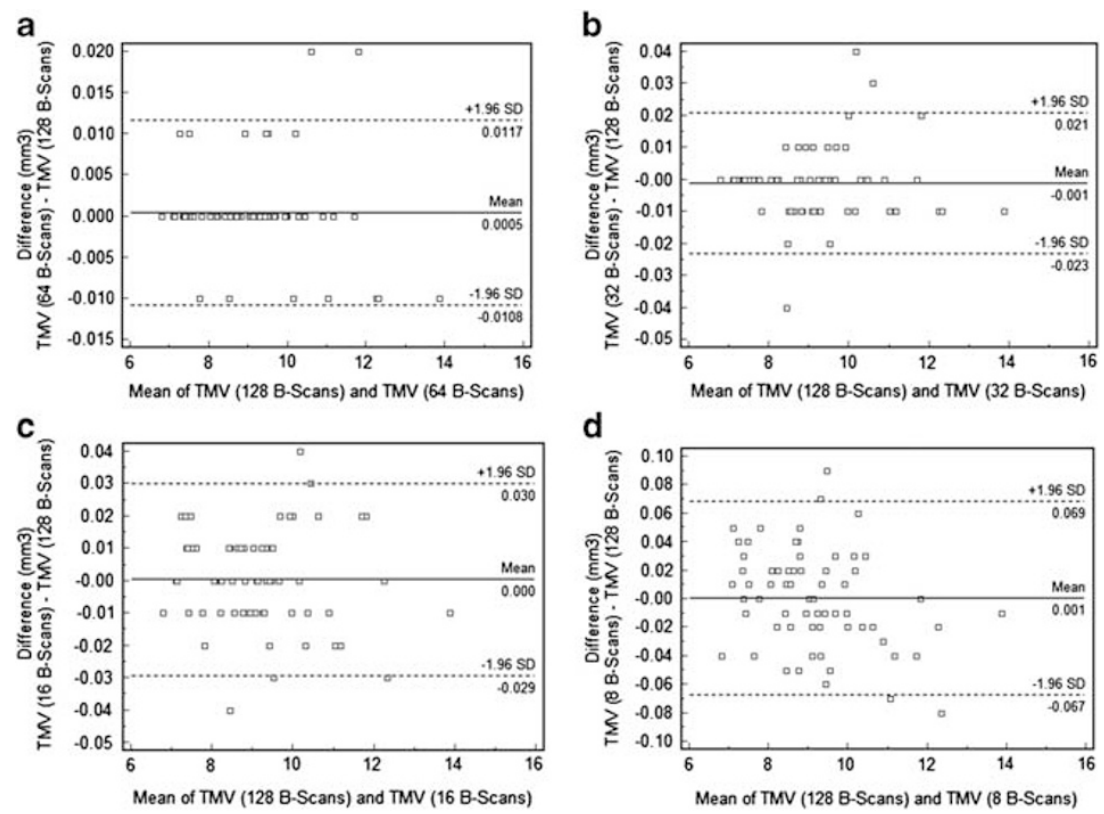

Figure 5 Bland-Altman plots demonstrating the level of agreement between the ground truth and each reduced sampling density for total macular volume $\left(\mathrm{mm}^{3}\right)$. Central solid line indicates the mean absolute difference and the dotted line indicates the $95 \%$ confidence interval limits (upper and lower). (a) At a B-scan-sampling density that includes every other B-scan (64 B-scans with an equal spacing of $94 \mu \mathrm{m}$ ). (b) At a B-scan-sampling density that includes every fourth B-scan (32 B-scans with an equal spacing of $188 \mu \mathrm{m}$ ). (c) At a B-scan-sampling density that includes every eighth B-scan (16 B-scans with an equal spacing of $376 \mu$ m). (d) At a B-scan-sampling density that includes every sixteenth B-scan (eight B-scans with an equal spacing of $752 \mu \mathrm{m}$ ).

Table 3 Corrected versus uncorrected foveal central subfield thickness and total macular volume values with differences between groups at each frame-sampling density

\begin{tabular}{|c|c|c|c|c|c|c|}
\hline B-scan density & Corrected & Uncorrected & Mean & $S D$ & Min & $\operatorname{Max}$ \\
\hline FCS RT $(\mu \mathrm{m})$ & & & \multicolumn{4}{|c|}{ Difference $(\mu m)^{\mathrm{a}}$} \\
\hline 128 & 345.17 & 343.23 & 7.06 & 17.83 & 0.00 & 115.70 \\
\hline 64 & 345.22 & 342.41 & 7.97 & 18.81 & 0.00 & 115.70 \\
\hline 32 & 345.02 & 342.66 & 8.05 & 18.99 & 0.00 & 119.70 \\
\hline 16 & 345.97 & 343.67 & 7.92 & 18.66 & 0.00 & 119.00 \\
\hline 8 & 345.26 & 343.02 & 7.80 & 19.40 & 0.00 & 125.10 \\
\hline 4 & 336.63 & 336.77 & 10.48 & 28.97 & 0.00 & 196.10 \\
\hline$T M V\left(m^{3}\right)$ & & & \multicolumn{4}{|c|}{ Difference $\left(\mathrm{mm}^{3}\right)^{\mathrm{a}}$} \\
\hline 128 & 9.15 & 9.15 & 0.72 & 0.77 & 0.00 & 3.57 \\
\hline 64 & 9.15 & 9.15 & 0.72 & 0.77 & 0.00 & 3.56 \\
\hline 32 & 9.15 & 9.16 & 0.72 & 0.77 & 0.00 & 3.58 \\
\hline 16 & 9.15 & 9.15 & 0.72 & 0.77 & 0.00 & 3.58 \\
\hline 8 & 9.15 & 9.16 & 0.73 & 0.78 & 0.00 & 3.63 \\
\hline 4 & 9.15 & 9.17 & 0.78 & 0.80 & 0.00 & 3.51 \\
\hline
\end{tabular}

Abbreviations: FCS, foveal central subfield; Max, maximum; Min, minimum; RT, retinal thickness; TMV, total macular volume. ${ }^{a}$ Absolute difference.

grading protocols, requiring only a reduced subset of B-scans to generate accurate retinal thickness maps increases the feasibility of manual correction of segmentation errors. This may be of importance as the OCT B-scans for over $50 \%$ of the eyes in this study had

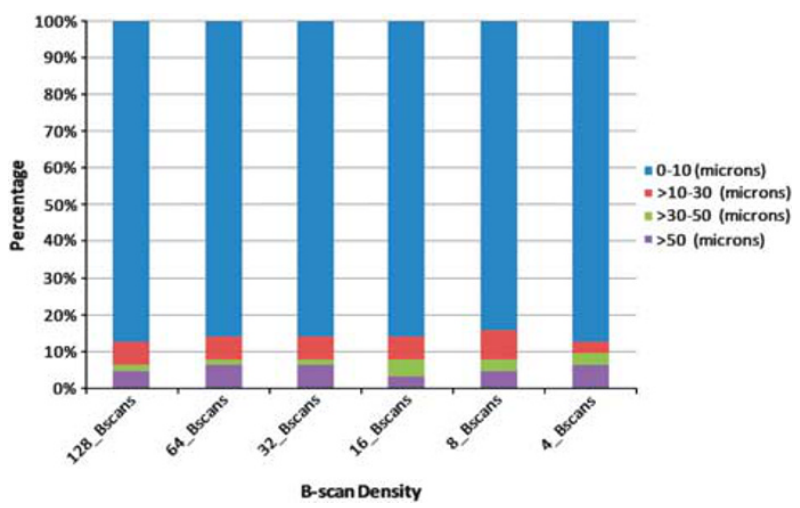

Figure 6 Frequency plot (cumulative percentage of cases) for difference in retinal thickness measurements between corrected and uncorrected groups. Difference measurements for the various plotted intervals are given in $\mu \mathrm{m}$.

evidence of segmentation errors that required manual correction.

On the other hand, we observed that correction of segmentation errors resulted in relatively small differences in retinal thickness and TMV measurements, regardless of the scan densities. Overall, only $12.5 \%$ of cases demonstrated a difference in FCS thickness of more than $10 \mu \mathrm{m}$ following manual correction.

Our study also has some limitations to consider when assessing the findings. First, only one SD-OCT 
instrument (Cirrus OCT) was used in this analysis. As the various instruments differ in their segmentation algorithms and may differ in the frequency and severity of segmentation errors, our findings regarding the need for manual correction may not generalize to other devices. Second, our study cohort is relatively small and included eyes with retinal thicknesses ranging from 187.7 to $705.4 \mu \mathrm{m}$. Thus, our findings may not extrapolate to eyes with even more severe edema. In addition, our analysis only focused on neurosensory retinal thickness. More localized parameters such as extent of subretinal fluid or cystoid spaces may require a greater density of scans for accurate quantification. Moreover, a greater scanning density may also be needed for qualitative morphological assessments such as identification of subtle areas of photoreceptor disruption. In addition, our study did not assess whether measurements would differ if even higher scan densities (>128 B-scans over $6 \mathrm{~mm}$ ) were used. Finally, high scanning density may also be critical for generation of OCT summation or projection maps, which are necessary for image-to-image and intervisit registration. Thus, high-density scans may still be needed, even if only subsets of these scans are used for quantification.

In summary, we observed that a B-scan density of only 16 equally spaced horizontal B-scans over a $6 \mathrm{~mm}$ square may be sufficient to adequately represent and generate a reliable macular retinal thickness map, regardless of whether segmentation errors are present. However, most scans include segmentation errors. Manual correction of OCT scans can address these segmentation errors, but only a minority of cases have clinically meaningful differences between corrected and uncorrected measurements. These findings may aid in the design of optimal SD-OCT scanning and analysis protocols for future studies of diabetic macular edema.

\section{Summary}

What was known before

- The previous reports on importance SD-OCT in diabetic macular edema assessment and management has been published.

\section{What this study adds}

- This paper for the first time evaluates the impact of scan density on spectral domain OCT (SD-OCT) thickness measurements in patients with diabetic macular edema. In particular, we define the minimum scanning density required in order to obtain reliable retinal thickness maps. Given that there are many SD-OCT instruments now available capable of performing volume scans of varying densities, we believe that our findings should be useful in the design of scanning protocols for clinical trials and clinical practice.

\section{Conflict of interest}

Dr Sadda also serves on the scientific advisory board for Heidelberg Engineering and receives research support from Carl Zeiss Meditec, Optos, and Optovue Inc.

\section{Acknowledgements}

This study was supported in part by NIH Grant EY03040 and an unrestricted grant from Research to Prevent Blindness, Inc. Drs Sadda and Walsh share in royalties from intellectual property licensed to Topcon Medical Systems by the Doheny Eye Institute.

\section{References}

1 Sánchez-Tocino H, Alvarez-Vidal A, Maldonado MJ, Moreno-Montañés J, García-Layana A. Retinal thickness study with optical coherence tomography in patients with diabetes. Invest Ophthalmol Vis Sci 2002; 43: 1588-1594.

2 Lim MC, Hoh ST, Foster PJ. Use of optical coherence tomography to assess variations in macular retinal thickness in myopia. Invest Ophthalmol Vis Sci 2005; 46: 974-978.

3 Sadda SR, Wu Z, Walsh AC. Errors in retinal thickness measurements obtained by optical coherence tomography. Ophthalmology 2006; 113: 285-293.

4 Ghazi NG, Kirk T, Allam S, Yan G. Quantification of error in optical coherence tomography central macular thickness measurement in wet age-related macular degeneration. Am J Ophthalmol 2009; 148: 90-96.

5 Hosseini H, Razeghinejad MR, Nowroozizadeh S, Jafari P, Ashraf H. Effect of macular edema on optical coherence tomography signal strength. Retina 2010; 30: 1084-1089.

6 Giani A, Cigada M, Esmaili DD. Artifacts in automatic retinal segmentation using different optical coherence tomography instruments. Retina 2010; 30: 607-616.

7 Patel PJ, Chen FK, da Cruz L, Tufail A. Segmentation error in Stratus optical coherence tomography for neovascular age-related macular degeneration. Invest Ophthalmol Vis Sci 2009; 50: 399-404.

8 Leung CK, Chan WM, Chong KK. Alignment artifacts in optical coherence tomography analyzed images. Ophthalmology 2007; 114: 263-270.

9 Ray R, Stinnett SS, Jaffe GJ. Evaluation of image artifact produced by optical coherence tomography of retinal pathology. Am J Ophthalmol 2005; 139: 18-29.

10 Glassman AR, Beck RW, Browning DJ, Danis RP, Kollman C, Diabetic Retinopathy Clinical Research Network Study Group. Comparison of optical coherence tomography in diabetic macular edema, with and without reading center manual grading from a clinical trials perspective. Invest Ophthalmol Vis Sci 2009; 50: 560-566.

11 Ahlers C, Simader C, Geitzenauer W. Automatic segmentation in three-dimensional analysis of fibrovascular pigment epithelial detachment using high-definition optical coherence tomography. Br J Ophthalmol 2008; 92: 197-203.

12 Legarreta JE, Gregori G, Punjabi OS, Knighton RW, Lalwani GA, Puliafito CA. Macular thickness measurements in normal eyes using spectral domain optical coherence tomography. Ophthalmic Surg Lasers Imaging 2008; 39: S43-S49. 
13 Keane PA, Mand PS, Liakopoulos S, Walsh AC, Sadda SR. Accuracy of retinal thickness measurements obtained with Cirrus optical coherence tomography. Br J Ophthalmol 2009; 93: 1461-1467.

14 Sadda SR, Keane PA, Ouyang Y, Updike JF, Walsh AC. Impact of scanning density on measurements from spectral domain optical coherence tomography. Invest Ophthalmol Vis Sci 2010; 51: 1071-1078.

15 Sadda SR, Joeres S, Wu Z. Error correction and quantitative subanalysis of optical coherence tomography data using computer-assisted grading. Invest Ophthalmol Vis Sci 2007; 48: 839-848.

16 Joeres S, Tsong JW, Updike PG. Reproducibility of quantitative optical coherence tomography subanalysis in neovascular age-related macular degeneration. Invest Ophthalmol Vis Sci 2007; 48: 4300-4307.

17 Sull AC, Vuong LN, Price LL. Comparison of spectral/ Fourier domain optical coherence tomography instruments for assessment of normal macular thickness. Retina 2010; 30: 235-245. 